\title{
Pudeur et liberté chez le traducteur Philéas Lebesgue
}

\author{
Émilie AUDIGIER \\ Universidade Federal de Santa Catarina \\ Brésil
}

\begin{abstract}
Résumé : Philéas Lebesgue (1869-1958), l'un des premiers passeurs de littératures « marginales » en France, transmet en langue française, au début du XX ${ }^{\mathrm{e}}$ siècle, des romans et nouvelles d'écrivains brésiliens et écrit de nombreuses chroniques dans des revues littéraires. En quoi son originalité intellectuelle le distingue des autres traducteurs de J osé de Alencar ou Machado de Assis ? Et comment son rapport à la morale en littérature s'exprime-t-il dans ses traductions ? Nous proposons d'analyser dans un premier temps son parcours d'érudit, que nous éclairerons ensuite par l'analyse d'extraits de ses traductions afin de comprendre ainsi l'usage qu'il fait de la « pudeur » en traduction.
\end{abstract}

Mots-clés : Philéas Lebesgue, traducteur français, littérature brésilienne, pudeur, liberté.

\begin{abstract}
Philéas Lebesgue (1869-1958), a leading intellectual of "peripheral" foreign literature in France, passed as a French Brazilian writer in early twentieth century and wrote many chronicles in literary journals. How is this original intellectual distinct from other French translators of J osé de Alencar and Machado de Assis? And most importantly, how is his rapport with morals in literature expressed in his translations? We propose to analyze at first his career as an erudite and to demonstrate with close readings of extracts from his translations how he is or is not "modest" in his translations.
\end{abstract}

Keywords: Philéas Lebesgue, French translation, Brazilian literature, bashfulness, freedom.

On associe la notion de pudeur en traduction aux Belles Infidèles, tendance historique qui, depuis le XVIIIe siècle, a marqué les traductions en France et en Allemagne, pendant le romantisme du XIX ${ }^{\text {e }}$ siècle jusqu'au $\mathrm{XX}^{\mathrm{e}}$ siècle. Ainsi, le «bien traduire» ne devait en aucun cas heurter les mœurs de l'époque et le lecteur éduqué, à la mode des salons littéraires, avait affaire à un type de héros prude et lissé, qui brillait alors en tant que modèle de parfait gentilhomme. On en trouve l'illustration chez la traductrice Mme de Staël, qui revisite les classiques gréco-latins en censurant et adaptant selon les critères de l'époque. La pudeur devient ainsi synonyme de morale, et la littérature, principalement sa dimension érotique, faisait l'objet d'une censure religieuse et sociale. La pudeur 
renvoie à la présence du traducteur et, par opposition, l'impudeur renverrait également à une présence du traducteur, qui n’hésite pas à accentuer l'intensité des figures de style et l'audace du lexique, sans omettre, ni dissimuler. Des caractéristiques que l'on rencontre chez les retraducteurs contemporains de Machado de Assis, qui rejettent de manière majoritaire cette tendance de traduction s'employant à embellir et à s'éloigner de la source, par pudeur ou par esprit créatif.

L'érudit Philéas Lebesgue contribua largement à la diffusion des Lettres portugaises et appartient à ce premier type de traductions que l'on qualifie de « pudiques ». Il collaborait régulièrement pendant près de 45 ans - de 1896 à 1940 - à la revue Mercure de France pour les rubriques des Lettres portugaises et brésiliennes, grecques, yougoslaves, (ainsi que norvégiennes, pendant une durée plus courte), littératures qui, à son époque, étaient pratiquement inconnues et marginales en France. D'une nature très curieuse, cet intellectuel était notamment le correspondant de plus de deux cent revues culturelles telles que Le Beffroi, La Phalange, La Vie, Les Mille Nouvelles Nouvelles, Águia, La Revue Bleue, La Revue de l'Amérique Latine etc.

Outre son activité de critique littéraire, il traduisit des livres de diverses langues. De la langue portugaise, il tradusit principalement des écrivains portugais (tel qu'Affonso Lopes Vieira), il fut notamment l'un des premiers à découvrir Fernando Pessoa en France. Du Brésil, il traduisit les écrivains J osé de Alencar, Iracéma en 1928 publié aux éditions Gedalge et deux romans avec son collaborateur et ami Pierre-Manoel Gahisto : Um rei Negro, de Coelho Neto ${ }^{1}$, publié au Brésil en 1914, sa traduction parut sous le titre de Macambira (le prénom de l'esclave noir, personnage principal du roman). Puis, en 1928, il traduisit Janna et Jöel de Xavier Ferreira Marques. Ces deux écrivains brésiliens, aujourd'hui moins connus et moins lus, car ils furent, dans le cours de l'histoire littéraire, amplement condamnés par les écrivains modernistes brésiliens qui leur succédèrent. Avec son collaborateur belge Manuol Gahisto (1878-1948) ${ }^{2}$, traducteur de l'italien, de l'espagnol, du portugais et du grec moderne, il réalisa un travail de traduction à quatre mains. Ensemble, ils publièrent, outre les deux romans brésiliens cités, une anthologie de nouvelles.

Passionné de langues, mais ayant quitté très tôt l'école, sa curiosité intellectuelle le conduisit à apprendre plus de seize langues étrangères, dont

\footnotetext{
${ }^{1}$ À propos de Coelho Neto, Philéas Lebesgue écrit: «Il possède la grâce dans le fantasme, l'exactitude dans l'abondance et sés descriptions peuvent se comparer à celles de Ramayana, par la richesse luxuriante de la couleur et de la vie qui y règnent.» (Mercure de France).

${ }_{2}^{2}$ Aussi appelé Paul Tristan Coolen, belge, il collabore aux revues Le Beffroi, La Province, La Revue d'Amérique Latine, traduit du portugaise trois autres romans Le Mulâtre, Macambira (1920) et J anna et J oel (1928) et un roman de l'italien Vierge Créole de G. Beccari (1920).
} 
les principales langues européennes (allemand, anglais, danois, espagnol, galicien, gallois, grec, norvégien, polonais, portugais, roumain, russe, serbocroate, slovène et tchèque), ainsi que des langues anciennes, telles que le sanskrit, l'ancien français et des langues régionales de France comme le breton, le provençal et le picard, lui-même étant originaire de NeuvilleVault, dans l'Oise.

Philéas Lebesgue traduisit notamment des romans de l'espagnol, L'évangile de l'amour de Gomez Carillo et À travers champs de Élie Lascaux, en 1952. En raison de sa passion pour les cultures étrangères, pour la lusophonie, il rêvait d'une unification de la culture portugaise et brésilienne, liées l'une à l'autre par leur langue commune et leur lien historique. Dans cette même perspective, il inaugura l'idée, novatrice au début du $\mathrm{XX}^{\mathrm{e}}$ siècle, de francophonie, nullement dans une perspective coloniale, mais plutôt en vertu d'une conception romantique et originale mettant en relation littératures africaines, antillaise et française via leur langue commune. En effet, s'il estimait que les littératures portugaises et brésiliennes n'étaient pas « complètement autonomes ([celles-ci] étant tributaires avant tout de la France devenue le cerveau latin) », il rejoint l'idée développée par Pierre Rivas ${ }^{3}$, selon laquelle le Brésil et les pays d'Amérique du Sud en général, pour s'affranchir de leur modèle colonial, ici le Portugal, et l'Espagne pour les autres pays, ont choisi la France comme modèle intellectuel afin de secouer le joug du pays colonisateur et de se constituer une identité propre. Une identité littéraire que Philéas Lebesgue commença à percevoir en montrant ses premiers contours au public français, dès les années 1910 et en en affirmant l'autonomie dans les années 1940.

Cet étonnant autodidacte possèdait une immense curiosité et créativité, dans le domaine des lettres comme dans celui du terroir. Fils d'agriculteur, activité qu'il exerçait en parallèle à son travail intellectuel, on l'appellait le «Virgile picard» en raison des poèmes qu'il écrivit sur sa région. En plus de ses activités littéraire, son engagement politique le conduisit, en militant laïque, à être maire de sa commune de Neuville pendant plus de trente-neuf ans. Dernier aspect de sa personnalité, il devint druide et maîtrisait les mystères de la nature et la langue bretonne. À ce sujet, il écrivit plusieurs livres ésotériques ${ }^{4}$.

Mais avant tout, Philéas Lebesgue était poète, auteur des recueils de poèmes ${ }^{5}$ Le buisson ardent (1910) et Les servitudes (1928), auteur de drames lyriques : Dieu et démon, publié en 1939, de tragédies : Le rachat de

${ }^{3}$ Rivas, Pierre. Diálogos interculturais. França : Portugal, Brasil. Brasília : Hucitec, 2005.

4 Parmi ses ouvrages ésotériques, nous notons Sous le chêne des druides, publié en 1931, en langue picarde « Ein acoutant l'Cloque de lToussaint».

${ }^{5}$ Le Buisson ardent, Éd. Henry Cormeau, (1910), Les Servitudes, Éd. du Mercure de France (1913), Présages, Éd. André Delpeuch, (1928) - Prix J ean Moréas en 1929. 
Prométhée, (1947), de romans : Le sang de l'autre (1901) ${ }^{6}$ et de nombreux essais sur la phonétique, la littérature et la formation du goût français. ${ }^{7} \mathrm{Il}$ décéda en 1958, peu connu, malgré une œuvre importante, constituée de milliers de chroniques littéraires publiées dans plus de 200 revues. Il reçut pour son travail de critique, traducteur et écrivain plusieurs récompenses : en 1929, le prix Moréas pour son recueil de poèmes Présages ; dix ans plus tard, un prix lui est accordé par l'Académie Goncourt. Plusieurs prix de l'Académie française lui furent également décernés : en 1920, le prix J ules Janin pour une traduction du grec moderne, puis, en 1956, le «prix de l'Académie française » pour son roman Mes Semailles. Il fut enfin promu chevalier de la Légion d'honneur en 1925, puis officier, en 1953.

Ces éléments biographiques esquissent les contours d'un personnage surprenant, aux multiples talents, pionnier dans la transmission de la culture lusophone en France. Il signait ses articles sous des pseudonymes aux consonnances étrangères : " Démétrius Astériotis » et «Lioubo Sokolovitchi ». Grand admirateur de l'écrivain portugais Aquilino Ribeiro, il fut aussi marqué dans sa jeunesse par le symbolisme, ainsi que par la littérature de Victor Hugo.

Ses choix de publication, son profil d'intellectuel, linguiste, critique et écrivain permettent d'évaluer ses tendances de traduction. Voici tout d'abord un commentaire de Machado de Assis, dans sa correspondance avec Figueiredo Pimentel (le 31 mars 1901), qui lui avait appris le projet de Philéas Lebesgue :

À Mr Figueiredo Pimentel. Je réponds à votre lettre, pour vous remercier des nouvelles que vous me donnez de Philéas Lebesgue, et de votre amabilité à faire en sorte qu'il traduise mes livres. Pourtant, je ne peux pas ordonner à l'éditeur de vous les envoyer, comme vous me le demandez, ni même d'autoriser la traduction, car la propriété de mes oeuvres a été transférée à Mr Garnier à Paris, avec tous les droits afferants. Seul lui pourra résoudre cette question. ${ }^{8}$

\footnotetext{
${ }_{6}^{6}$ Le Sang de l'autre (1901), Compiègne : réédition Éd. Le Trotteur ailé, 2010 ; La Nuit rouge, Éd. Sansot, 1905; Terre picarde, Éd. du Bonhomme picard, 1950.

7 « Les Lois de la parole, essai de synthèse phonétique », Imp. du Moniteur de l'Oise (1899) ; « La Grèce littéraire d'aujourd'hui », Éd. Sansot (1906) ; « L'Héritage intellectuel de Virgile au Portugal et en France», Éd. Institut français au Portugal (1932); Aux Fenêtres de France: Essai sur la Formation du Goût Français, Paris : Éd. Garniers, 1906.

8 « Ex.mo Sr. Figueiredo Pimentel. / Respondo à sua carta, agradecendo as notícias que me dá relativamente a Philéas Lebesgue, e à solicitude de V. Ex.a em fazer com que este traduza os meus livros. Entretanto, não posso ordenar que o editor lhos envie, como V. Ex.a me pede, nem sequer autorizar a tradução, porquanto a propriedade das minhas obras está transferida ao Sr. Garnier, de Paris, com todos os respectivos direitos. Só ele poderá resolver sob esse ponto. / Sou, com apreço e consideração, de V. Ex., at. venerador e obrg. Machado de Assis. »
} 
(http:/ / www.portalsaofrancisco.com.br/alfa/ centenario-de-machado-deassis/ correspondencia-3.php)

Ce commentaire montre que Philéas Lebesgue, en correspondance avec de nombreux écrivains du monde entier', avait l'ambition de traduire Machado, lequel ne connaîtra de son vivant aucune traduction française, ayant été publié en français pour la première fois en 1910 par Lebesgue, dix ans après cet échange de lettres dans la revue Mille nouvelles nouvelles.

Voici également un commentaire d'Anatole France, qui, en 1910, lors de la « fête de l'intellectualité brésilienne [...] dont le but principal était de rendre hommage à l'écrivain Machado de Assis, décédé l'année précédente, et de diffuser ses œuvres en France », déclara :

Grâce à Luiz Annibal Falcão qui a cherché à adapter chaque texte plutôt qu'à le traduire, et grâce aussi à $M$. Manuel Gahisto, qui s'est particulièrement intéressé au texte de « Page relue », les lecteurs français pourront prendre un premier contact avec des auteurs dont l'œuvre, nous l'espérons, ne décevra pas. (in Orban 1917, 19)

En effet, en 1917, la traduction de Lebesgue propose une interprétation personnelle quant à la vision et la transmission de l'œuvre de Machado. Certes, le contexte littéraire de l'époque influence et change la lecture que l'on fait de son œuvre. On remarque ici que l'« adaptation » plutôt que traduction revêt un sens positif. Soucieux de la réception du lecteur français et prenant en compte le type de public pour lequel il traduit, Philéas Lebesgue opère plusieurs changements dans le texte, le « lissant ».

On rencontre cette même préoccupation dans sa traduction du roman Iracema d'Alencar, comme le souligne deux spécialistes français de la littérature brésilienne, J ean-Michel Massa et Marie-Hélène Torres $^{10}$, il utilise l'expression «jeune fille» pour «virgem», afin de minimiser l'aspect érotique trop présent pour Lebesgue, lorsque la retraductrice, sept décennies plus tard, utilise le terme «jeune vierge». Par ailleurs, il préfère censurer les passages du texte où l'érotisme et la sensualité sont au centre du propos. Il coupe ainsi les passages amoureux entre l'héroïne Iracema et le héros :

\footnotetext{
9 Citons sa correspondance avec les Français Claude Aveline, Georges Duhamel, Édouard Dujardin, Émile Guillaumin, Pierre J ean J ouve, Louis Pergaud, Henri Pourrat, Émile Verhaeren, René Maran de Martinique, Constantin Balmont de Russie, F. T Marinetti d’Italie, Oscar V. de L. Milosz de Lituanie, Anéglos Sikelianós de Grèce, Milan Voukassovitch de Serbie, Bernardino Machado du Portugal, Manuel Teixeira Gomes et Theophile Braga du Portugal.

${ }^{10}$ Cf. Références bibliographiques.
} 
Agora podia viver com Iracema, e colher em seus lábios o beijo, que ali viçava entre sorrisos, como o fruto na corola da flor. Podia amá-la, e sugar desse amor o mel e o perfume, sem deixar veneno no seio da virgem.

(Iracema, 1865)
Et aussitôt son œeil frémit. Maintenant, il pouvait (Philéas Lebesgue, 1928) vivre avec Iracéma et cueillir dans ses lèvres le baiser qui s'y épanouissait entre les sourires comme le fruit dans la corolle de la fleur. Il pouvait l'aimer et humer de cet amour le miel et le parfum sans laisser le poison dans le sein de la vierge.

(Oséki-Dépré, 1985)

Les lèvres, le baiser, le parfum et le sein disparaissent dans la traduction de Lebesgue pour ne devenir qu'un « frémissement [de] l'œil ». Cette pudeur révèle encore une fois sa préoccupation à écrire pour être lu notamment par un jeune public. Elle révèle comment son idéologie et ses goûts transparaissent dans ses traductions : sa tendance au symbolisme et au romantisme, liée à la glorification de la nature (comme chez J osé de Alencar), quel'on retrouve dans sa propre production poétique.

Un autre passage montre encore une fois un trait idéologique qui renvoie à ses convictions politiques et religieuses. En effet, la question de la foi, présente chez J osé de Alencar, est aussi traitée de manière singulière par Philéas Lebesgue, athée et républicain, comme nous l'avons évoqué auparavant.

O cristão repeliu do seio Le jeune Portugais Le chrétien repoussa loin a virgem indiana. Ele repousse la vierge de son sein la vierge não deixará o rasto da indienne. Il ne laissera indienne. Il ne laissera desgraça na cabana point la trace du malheur pas de trace de malheur hospedeira. Cerra os dans la cabane dans la hutte hospitalière. olhos para não ver; e hospitalière. Il ferme les Il ferme les yeux pour ne enche sua alma com o yeux pour ne pas voir, et nome e a veneração e l'enseignement de sa seu Deus;

- Cristo !... Cristo!... $\quad$ (Iracéma, mère remonte à son âme. (Iracema, 1865) Lebesgue, 1928) Philéas pas voir; et il remplit son âme du nom et de la vénération de son Dieu; Christ!.... Christ!..

(Iracéma, Oséki-Dépré, 1985)

Ces changements, notamment mentionnés par J ean-Michel Massa, s'expliquent par la même volonté d'adaptation pour un public d'enfants. Ils reflètent donc la position idéologique du traducteur : Philéas Lebesgue en tant que républicain athée laïcise le texte en substituant «[cristão] » « chrétien », par « le Portugais », mettant accent sur la nationalité et non sur la religion, et [Cristo, Cristo] « Christ, Christ » par la périphrase: « il 
murmure des paroles de religion ${ }^{1}$, paroles qui explicitent et introduisent le regard du traducteur et la distance idéologique face au texte traduit. Il s'agit cette fois d'une pudeur quant à la question religieuse.

Il est certes aisé de condamner l'intervention du traducteur, mais il importe de prendre en considération l'idéologie de l'époque, et de reconnaître le mérite de Lebesque d'avoir traduit pour la première fois des écrivains brésiliens jusqu'alors inconnus en France. De fait, celui-ci procède à une adaptation conformément aux critères d'une époque, où les traducteurs adaptent plus qu'ils ne traduisent. Lorsqu'il supprime la partie de la phrase «la vénération de son Dieu» et la remplace par « l'enseignement de sa mère », le traducteur supprime la distance qui existe entre la culture de l'Indienne et celle du Portugais. La censure moralisante se manifeste notamment dans le fait qu'il remplace systématiquement le substantif «vierge» par la «jeune fille», laissant pudiquement l'implicite révéler ce que le texte original disait en toutes lettres. Les adaptations qu'opère Lebesgue dans les traductions de J osé de Alencar se retrouvent aussi dans celles de Machado de Assis.

D’après Claúdio Veiga $(1992,49)$, dans son livre Um brasilianista francês - Philéas Lebesgue, Lebesgue définit Machado de Assis comme un écrivain qui «détonne de la littérature du pays en échappant au déterminisme local $»^{2}$, lui reprochant son absence d'exotisme, « le manque de pittoresque et d'énergie. Il perçoit la vérité humaine et se désintéresse de la nature. ${ }^{3}$ (Veiga 1992, 34) Le terme «vérité humaine» de Philéas Lebesgue renvoie à un Machado "anthropologue», plus qu'à un auteur brésilien défendant les valeurs locales et la nature exubérante, qui attire tant les voyageurs de l'Ancien monde. Effectivement, Machado ne décrit pas, à la manière d'un Alencar indianiste, les charmes d'un Brésil luxuriant et en cela éloigné de la vieille Europe. Pourtant, ce défaut pointé par Lebesgue - ce « manque de pittoresque » - se révèle de fait une qualité, comme l'écrira plus tard Roger Bastide, qui prend la relève de Philéas Lebesgue à la revue Le Mercure de France pour la rubrique des Lettres brésiliennes à partir de 1949. Celui-ci voit en Machado de Assis un "paysagiste», mais les paysages évoqués ne sont pas purement géographiques, faits des noms de rues, de quartier, et résiderait avant toute chose dans la langue, novatrice et brésilienne, qu'il inaugure.

En cela, notons que la perception des traductions de la littérature brésilienne, a irrémédiablement changé en l'espace de plusieurs décennies. Comme la « couleur locale » d'un J orge Amado et le romantisme indianiste d'un J osé de Alencar séduit le lecteur français, Machado de Assis lui aussi

${ }^{1}$ Apud. Massa $(1971,53)$ et Torres $(1999,211)$.

2 « Ele destoa da literatura do país por escapar ao determinismo local. »

3 « O que se pode censurar em Machado de Assis é a falta de pitoresco e mesmo de energia.

Percebe a verdade humana e se desinteressa da natureza. » 
décrit l'esprit brésilien à travers le portrait de la société de la fin du XIX siècle au moyen d'une langue sophistiquée, teintée d'une omniprésente et subtile ironie.

Cette subtilité, Philéas Lebesgue est loin de l'avoir omise, bien au contraire, a tendance à l'exacerber. Prenons ce passage de la nouvelle «L'infirmier » où le personnage principal est injurié par son patron, le colonel qu'il est chargé de soigner (Cf. Audigier 2010). Encore une fois, il apporte une touche personnelle au texte.

Era burro, camelo, J'étais un mulet, Bourrique, pedaço d'asno, un chameau, un chameau, idiota, moleirão, âne, un idiot, un idiot, balourd : era tudo. Nem, ao lourdeau, tout, j'étais tout. Pour menos, havia mais enfin. Et personne comble, il n'y avait gente que pour venir plus personne qui recolhesse uma partager avec moi recueillit une part parte desses la série de ces de ces doux nomes. (Machado épithètes. de Assis 1896, 132)

[...] j'étais âne bâté, chameau, ignorant, idiot, lourdaud, j'étais tout ! Encore fautil savoir que j'étais seul à recevoir tous ces noms. (Orban 1914, 142) (Lebesgue 1911, 122)

«Mulet ou bourrique ou âne bâté», «idiot ou ignorant», « lourdeau ou balourd », ces nuances s'équivalent. La nuance réside surtout dans la dernière phrase : « uma parte desses nomes » pour « la série de ces épithètes » $(\mathrm{AD})$ ou « une part de ces doux vocables » (PL), ou simplement «ces noms » (VO). Les doux vocables rendent ici l'ironie toute machadienne, ce que ne font pas Orban ni Delpech.

Reprenons l'exemple et l'analyse de la comparaison des traductions d'un passage de «L'infirmier » [ «O enfermeiro »], après que Procópio a tuéle colonel, et l'intervention d'un esclave.

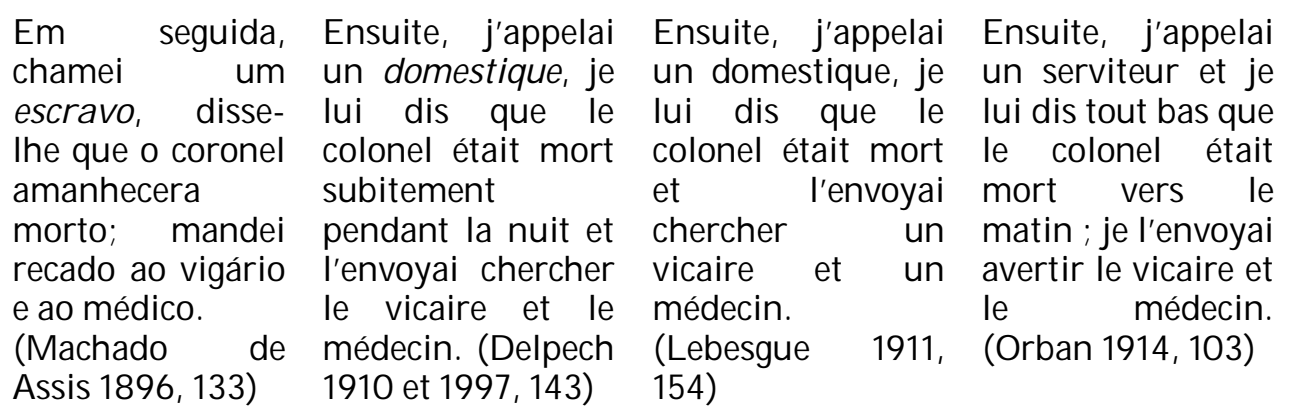


Pour traduire le mot « esclave », réalité contemporaine de Machado de Assis, tous les traducteurs français emploient, par pudeur, un terme plus faible, tels que «domestique» ou «serviteur», euphémismes visant à amoindrir la réalité historique. La réalité choquante de l'esclavage, que la France avait déjà aboli, paraît insupportable au point que tous les traducteurs censurent systématiquement, adaptant le texte de Machado aux valeurs républicaines françaises.

Ainsi, si, en comparaison avec les premières traductions de Machado de Assis des années 1910, Philéas Lebesgue s'autorise de nombreuses libertés, parfois poussées par une créativité lyrique qui caractérise son ouvre poétique singulière, d'autres fois il semble faire preuve de réserve surtout lorsqu'il s'agit de la question religieuse. Peut-on pour autant condamner cette marque personnelle de retenue, que les retraducteurs, en adéquation avec l'idéologie de leur époque, rectifieront ? Même s'il est vrai que Philéas Lebesgue, dans son travail de traducteur, modifie le texte, peut-on envisager cette « pudeur » du traducteur comme étant aussi en accord ou en rupture avec l'esprit de l'époque. Autrement dit, en découvrant des écrivains qui jusqu’à présent étaient ignorés en France, en choisissant de traduire des auteurs de langues dites « rares » et oubliées, Philéas Lebesgue fait preuve d'une audace digne des esprits les plus éclairés.

« Eça de Queiroz, Machado de Assis, Anatole France, trois esprits de la même famille », affirme-t-il, non sans une tendance à associer par goût pour l'universalisme trois écrivains qui, selon lui, avaient comme point commun la latinité. Latinité commune indéniable, malgré les inspirations d'origine africaine et indienne de la littérature brésilienne qu'il ne laissera pas pour compte (traduisant les romans Iracema, une légende indienne et Um rei Negro). En outre, dans le contexte idéologique de son époque, il a montré autant d'audace, voire davantage, qu'un traducteur qui, dans les conditions actuelles, ne supprimerait pas les scènes érotiques, ou les passages litigieux, suivant le goût du public contemporain. En cela, la liberté de Philéas Lebesgue est infiniment audacieuse.

\section{Références bibliographiques}

Alencar, J osé Martiano de. Iracéma, trad. Philéas Lebesgue et Pierre-Manoel Gahisto. Paris : Édition Gedalge, 1928.

Alencar, J osé Martiano de. Iracéma, Légende du Céara, trad. Inês Oseki-Dépré. Aix-en-Provence : Alinéa et Paris : Édition Unesco, 1985.

Antunes, Benedito. Sérgio Vicente Motta. Machado de Assis e a crítica internacional. São Paulo: Unesp, 2009.

Audigier, Émilie. Les traductions françaises de Machado de Assis et Guimarães Rosa : variations de huit nouvelles de 1910 à 2004. Thèse de doctorat. Université de Provence et UFRJ , 2010. 
Ferreira Cunha, Eliane Fernanda. Para Traduzir o século XIX: Machado de Assis. São Paulo : Anna Blume, Academia Brasileira de Letras, Selo Universitário, 2004. France, Anatole. Machado de Assis et son œeuvre littéraire. Paris : Édition Louis Michaud, 1909.

Machado de Assis. «L'infirmier». In: Mille Nouvelles Nouvelles $\mathrm{n}$ o 14 «La Renaissance du livre». Traduit par Philéas Lebesgue et Manoel Gahisto. Paris : mars 1911: 97-104.

Machado de Assis. "L'infirmier». In: Várias histórias. Traduction d'Adrien Delpech. Paris : Édition Garnier Frères, 1910, épuisé.

Machado, une Euvre littéraire, une vie. Traduction de Victor Orban. Paris: Édition Garnier Frères, 1917.

La Cartomancienne et autres histoires. Traduction d'Adrien Delpech. Toulouse : Del'Ombre, Petite Bibliothèque Ombres, 1997.

Massa, J ean-Michel. « Iracéma en Picardie ». In : Nouvelles Études Portugaises et Brésiliennes, Travaux de l'Université de Haute Bretagne, p. 31-35.

Massa, Jean-Michel. Machado de Assis Traducteur. Poitiers (tese de doutoramento, mimeografada), s.d.

Mounin, Georges. Les Belles Infidèles. Paris : Éditions Cahier du Sud, 1955.

Orban, Victor. Littérature Brésilienne, traduction de Victor Orban, préface de M. de Oliveira Lima. Paris : Éditions Garnier, s.d.

Orban, Victor. Machado de Assis, son œeuvre littéraire. Avant-propos d'Anatole France. Paris : Éditions Garnier, 1917.

Orban, Victor. Poésie Brésilienne, trad. de Victor Orban, préface de Pimentel Brandão. Paris : Éditions Garnier, 1922.

Quelques Conteurs Brésiliens. Édition établie par l'Académie Brésilienne de Lettres, trad. de Luiz Anibal Falcão, préface de Cláudio de Souza. Paris: Éditions du Sagittaire, 1938.

Rivas, Pierre. Diálogos interculturais. França : Portugal, Brasil. Brasília : Hucitec, 2005.

Staut, Lea Mara Valesi. A Recepção da obra machadiana na França. In: Mariano, Ana Salles Oliveira, Maria Rosa Duarte de (Org.). Recortes Machadianos. São Paulo: EdUC, FAPESP, 2003.

Staut, Lea Mara Valesi. Machado e Brás Cubas na França. Revista de Letras, São Paulo, n. 29 (1989) : 71-77.

Staut, Lea Mara Valezi. O Estilo Machadiano e o tradutor. Alfa : São Paulo, n. 36 (1992) : 11-117.

Staut, Lea Maria Valezi. Traduções Francesas da obra machadiana. Trabalhos em Lingüística Aplicada, Campinas, n. 24, p. 35-43, jul./ dez. 1994.

Torres, Marie-Hélène. Variations sur l'Étranger dans les lettres: cent ans de traductions françaises des lettres brésiliennes. Arras : Artois Presses Université, 1999.

Veiga, Claúdio. Um Brasilianista Francês. Rio deJ aneiro: Topbooks, Fundação Cultura do Estado da Bahia, 1998. 